\title{
A Simulation and Measurement on the Unintentional Emissions for a Power Converter Module
}

Gyoo-Soo Chae*

Division of Information and Communication Engineering, Baekseok University, Korea; gschae@bu.ac.kr

\begin{abstract}
Background/Objectives: An integrated circuit enabled the manufacturing of small electronic appliances. However, these circuits were vulnerable to unwanted emissions from various power sources. We present an investigation study on EMI (Electromagnetic Interference) of a power converter circuit for solar energy systems. Methods/Statistical Analysis: We use an artificial network to estimate and measure the unintentional emissions on power lines. The components normally act as an isolation circuit. A DC-DC power converter circuit is designed and manufactured. The simulation results of unintentional emissions are presented. To reduce the emissions, a revised circuit with capacitors and ground posts is fabricated. Test methods and conditions used here are explained. Findings: The emissions for conduction and radiation were measured in the EMI test facility based on the standardized testing procedures. The results show the unwanted emissions from the circuit are diminished tremendously due to the suggested EMI reduction techniques. Improvements/ Applications: In this study, the measured EMI emission values for a power converter circuit are presented. The results proposed here can be usefully applied on designing power converter modules.
\end{abstract}

Keywords: EMI, Emissions, Power Converter, Solar Energy

\section{Introduction}

In the 1970s, a logic circuit was very popular and its use grew rapidly and the switching speeds increased tremendously. And the integrated circuit enabled the manufacturing of small electronic appliances that allowed extremely low power consumption. These kinds of circuits consumed far less power were vulnerable to emissions from unexpected sources. Therefore, EMC (Electromagnetic Compatibility) incorporated into the circuit design is needed to work satisfactorily in commercial systems and modules. Recently, LSI (LargeScale Integration) chip components are popularly using and needed to reduce their operating voltage for small energy consumption. This results in maximized spurious emissions for electronic devices and modules to EMI. Unintentional electromagnetic sources generating power to electronic modules and circuits have become fascinating study topics. In this study, we present the investigation on an equivalent circuit method. These methods can be adapted to a wide range of complicated EMI problems. The acceptable level of EMI generated by an electronic device is strictly controlled by regulatory statute ${ }^{1}$. EMC Standards for electronic systems provide the typical conditions for the measurement methods and processes of the unintentional emissions. CISPR (International Special Committee on Radio Interference) is categorized by applications. CISPR 22 deals with all types of Information Technology Equipment (ITE) ${ }^{2-4}$. It is applicable for compliance testing of systems at the certified test sites. We investigate a DC-DC power converter module for solar energy system. Normally, the flow of electric energy in a power converter is controlled based on a load demand. The conducting line and EMI filters are crucial parts of a converter system ${ }^{5}$. A power converter circuit is designed and measured the interference levels for the typical and optimized circuit $\frac{6,7}{}$.

${ }^{*}$ Author for correspondence 


\section{Theoretical Background of EMI}

Conductors on the electronic circuit carrying current at the certain frequencies may efficiently radiate electromagnetic energy. Therefore the current that is occurred on the conductor is crucial to understand on the radiations. In here, two types of modes that can exist on the electronic circuit will be studied. Currents with noise can be identified into two types: Differential mode and common mode. Noise generated on the circuit is added at the signal source and flows with current in differential mode. Figure 1 shows the differential mode noise and the suppression method 8 . In common mode, noise is added at the signal source and can effect on all conducting lines in the

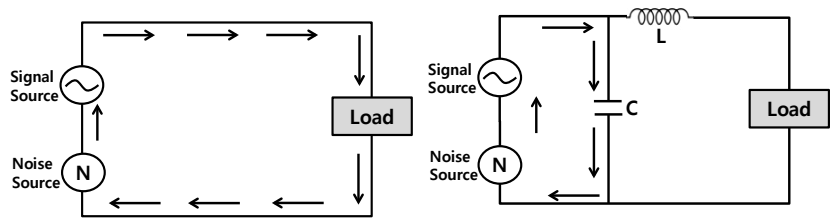

Figure 1. Differential mode noise and noise reduction circuit $\underline{6}$.
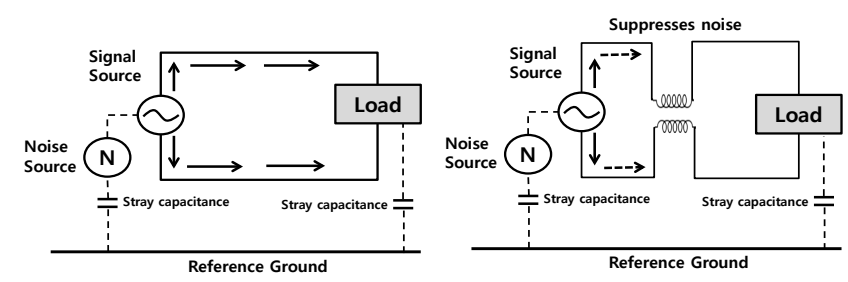

Figure 2. Common mode noise and noise reduction circuit ${ }^{6}$.

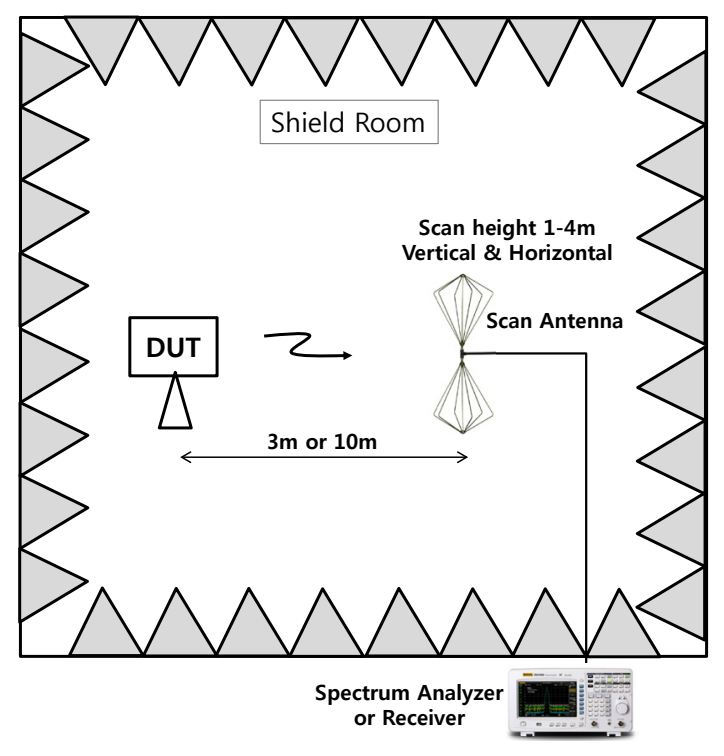

Figure 3. A shielding room and test setup for EMI. same direction. The source signal flows well through the inductor and noise is conducted to the ground line due to the frequency differences. Figure 2 shows the common mode noise and the method to diminish the noise effect. Noise is reduced by installing inductors to the signal line and ground line, respectively. FCC provides very detailed radiating emissions testing standards ${ }^{9,10}$. Radiating emissions are to be tested at the specific ranges of $3 \mathrm{~m}$ and $10 \mathrm{~m}$ depending on the devices specified in the standard. The measured results can be checked using standard dipole or antennas in vertical polarization and horizontal polarization. These measurements (FCC and CISPR 22) for the commercial products are to be done in a shielded semi-anechoic EMI chamber with electromagnetic wave absorbing structures on the walls $\frac{11,12}{12}$. Figure 3 shows a structure and testing setup for the shielding room.

\subsection{Conducted Emissions for Components/ Modules}

Unwanted emissions on power lines could be tested using a manufactured network. The artificial components act as an isolation circuit. The emissions from control lines and signal wires could be tested by a test probe. This can also affect to the measurements of the radiated emissions. Basically, radiated emissions are mainly the results from the conducting wire. Figure 4 presents the conducted emissions limits for FCC and CISPR 22. In CISPR 22, voltage measurements and current probe measurements are introduced. Voltage measurements for power lines on the converter circuit could be made relative to the DUT box. In case of the DUT with return paths distantly grounded, the voltage tests can be done on each signal line on the ground. The test equipment conducting wire would be placed at least $50 \mathrm{~mm}$ above the ground as specified in CISPR 22. The measurement using current probe could be done on the control lines and signal leads. It is normally compatible with the actual size of the probe for current measurement. The length of the test harness might be nominally $1.5 \mathrm{~m}$, spaced $50 \mathrm{~mm}$ above the ground surface as specified in CISPR 11. The test harness conducting wires would be typically parallel and adjacent. The current probe should be spaced $50 \mathrm{~mm}$ from the DUT connector. The conducted emissions are also measured using the same probe. In general, the peak values will be occurred as close to the DUT connector as possible. 


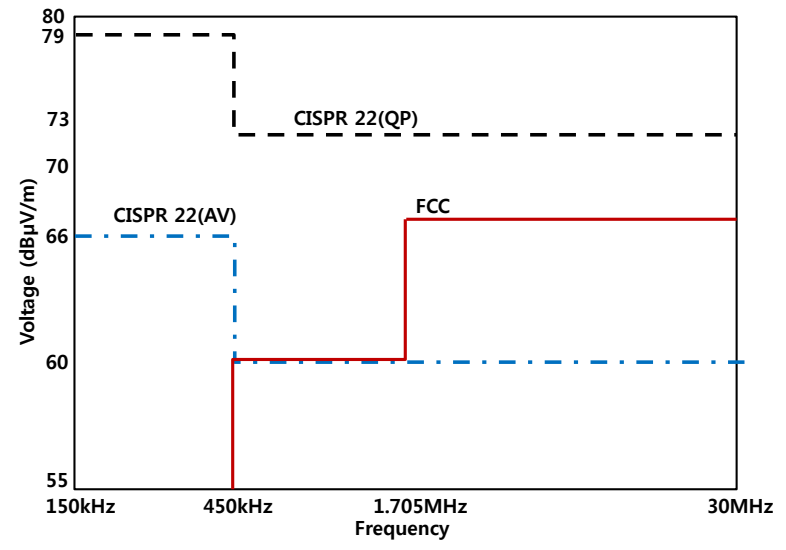

(a)

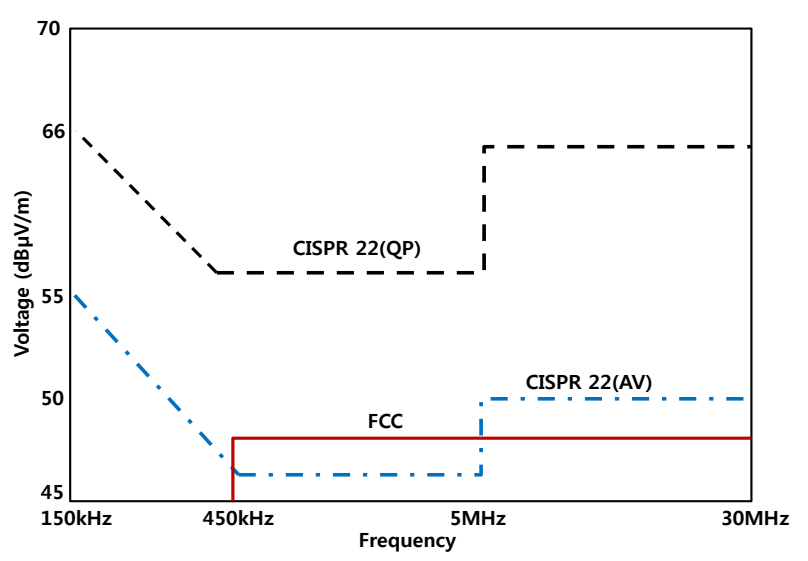

(b)

Figure 4. Conducted emissions limits for FCC and CISPR $22^{3}$. (a) For Class A devices, (b) For Class B devices.

\subsection{Radiated Emissions from Component/ Module}

All the electronic circuits connected to power radiate the electromagnetic waves in various frequency bands that unintentionally propagate away from the devices $\frac{13,14}{}$. The levels of the permissible radiated emissions are strictly regulated. In close proximity, large amounts of radiated emissions may interfere with the normal running of other systems. Therefore, it is very important to know about the origins of radiated emissions. The electronic circuit design can be considered to minimize the unwanted emissions. The circuit is used in this study is investigated to predict the emissions and reduce those. CISPR 22 standards demand that radiated emission tests for Class A modules can be done at a distance of $30 \mathrm{~m}$. Class B devices require that the emissions should be measured at a distance of $10 \mathrm{~m}$. It is noted that Class A devices are for a commercial, industrial or business environment. Class B devices should be used in a residential environment $t^{15}$. Figure 5 presents the radiated emissions limits for FCC and CISPR 22. Figure 6 shows the schematic of the presented converter using LT 337. Figure 7 shows the designed circuit with 1-layer PCB applied no EMI reduction techniques. Figure 8 shows the designed 2-layered PCB with ground posts put capacitors and ground posts to diminish the unwanted emissions. Figure 9 shows the manufactured 2-layered PCB with multiple ground posts.

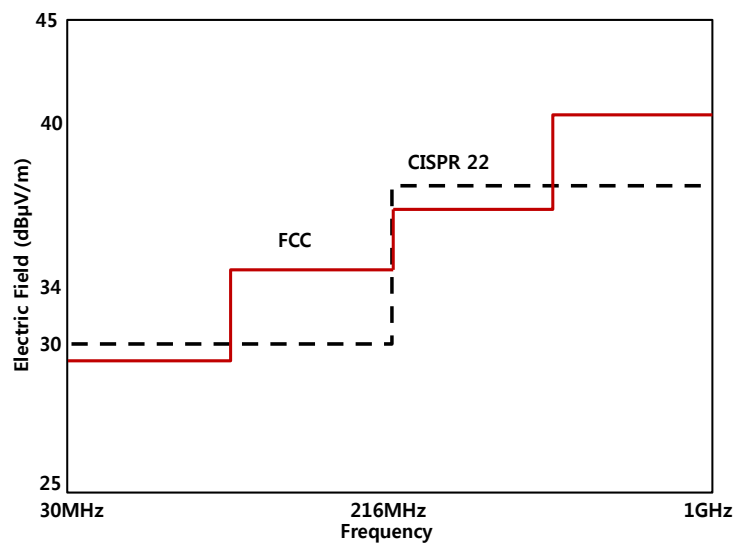

(a)

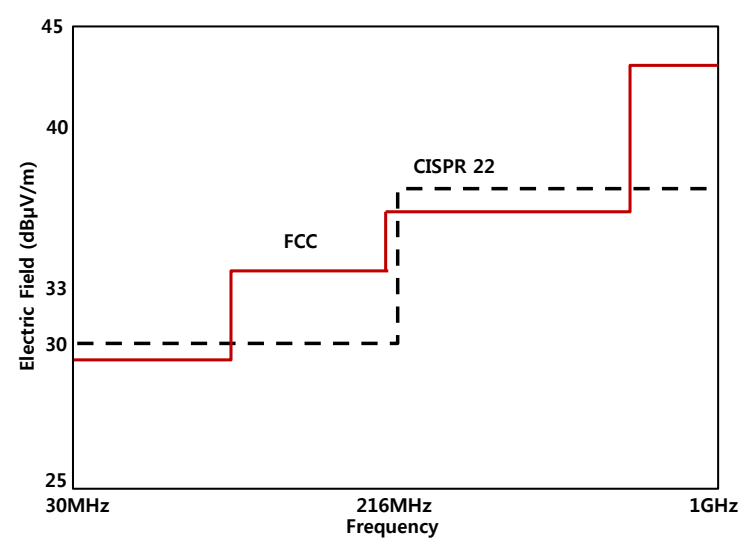

(b)

Figure 5. Radiated emissions limits ${ }^{3}$. (a) For class A devices, (b) For class B devices.

\section{Measurements of Emissions}

Conducted emission and radiated emission were measured as the suggested in the CISPR 22 (KN 22). The standard for Class A is applied for this measurement at the main power port. The limits for conducted emission are presented in Table 1. The limits for radiated emissions are presented in Table 2 and Table 3. Both present the limits for Class A devices. Figure 10 and Figure 11 shows the 


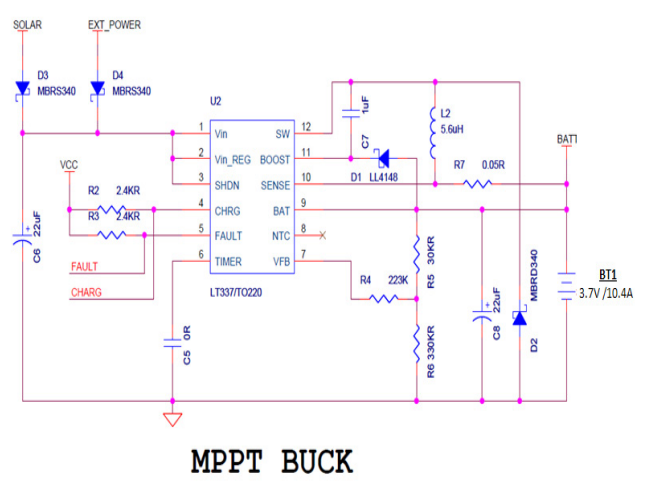

Figure 6. The schematic of the presented converter.

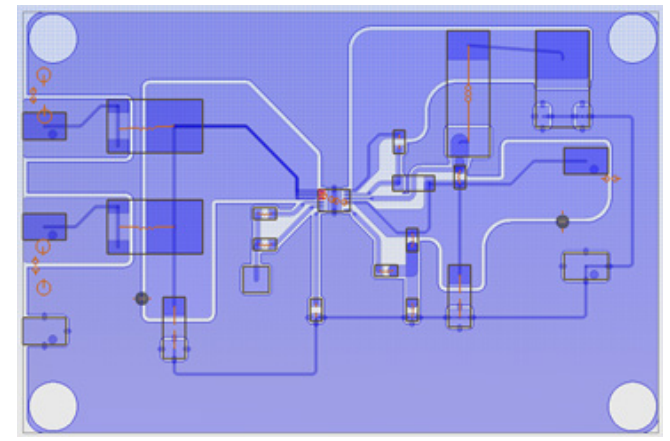

Figure 7. The designed circuit with 1-layer PCB.

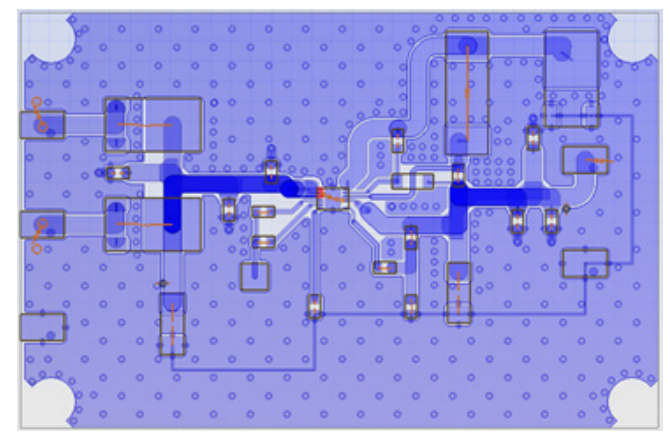

Figure 8. The designed 2-layered PCB with ground posts.

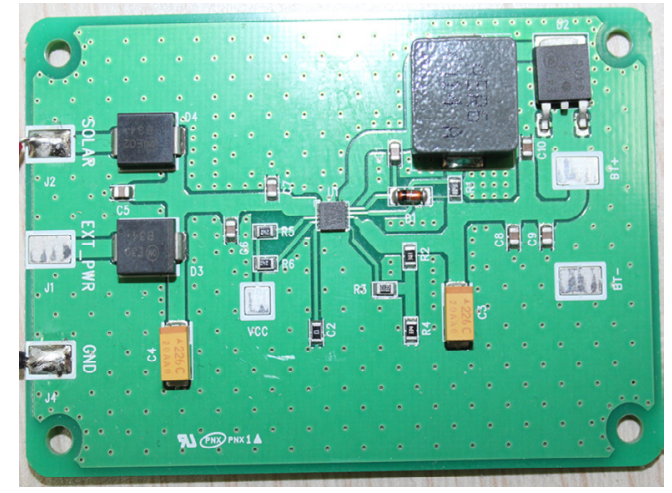

Figure 9. The manufactured 2-layered PCB with ground posts. 1-layer near field mode $\mathrm{H}$-field and E-field. The emissions are $1.12 \times 10^{3}[\mathrm{~A} / \mathrm{m}]$ and $1.44 \times 10^{2}[\mathrm{~V} / \mathrm{m}]$. Figure 12 and Figure 13 shows the 2-layer near field mode test for $\mathrm{H}$-field and E-filed before applying EMI reduction techniques. Figure 14 and Figure 15 shows the 2-layer near field mode test for $\mathrm{H}$-field and E-filed after applying EMI reduction techniques. The emissions are decreased radically to $8.9 \mathrm{x}$ $10^{2}[\mathrm{~A} / \mathrm{m}]$ and $1.0 \times 10^{2}[\mathrm{~V} / \mathrm{m}]$ due to the capacitors and the ground posts. Figure 16 shows CISPR-22 conducted RF Emissions with positive and negative voltage on supply lines. Figure 17 shows the test setup for CISPR-22 conducted RF emissions measurement. Figure 18 shows CISPR-22 radiated RF emissions with horizontal and vertical antenna position. Both results give a radiated emission levels under the specification. Figure 19 shows the test setup for CISPR-22 radiated RF emissions measurement.

Table 1. The limits for conducted emissions ${ }^{1}$

\begin{tabular}{|l|l|l|}
\hline Frequency $[\mathrm{MHz}]$ & \multicolumn{2}{|c|}{ Limits $[\mathrm{dB}(\mathrm{uV})]$} \\
\hline & Quasi-peak & Average \\
\hline $0.15-0.5$ & 79 & 66 \\
\hline $0.5-30$ & 73 & 60 \\
\hline
\end{tabular}
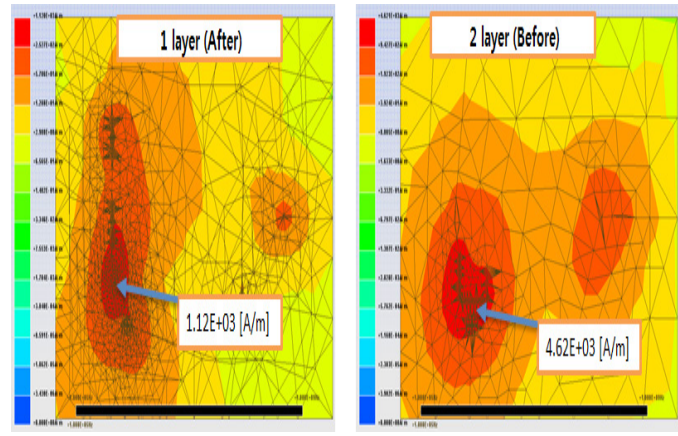

Figure 10. 1-layer near field mode test (H-field).

Table 2. The limits for radiated emissions for below 1 $\mathrm{GHz}^{1}$

\begin{tabular}{|l|l|}
\hline Frequency $[\mathrm{MHz}]$ & Quasi-peak $[\mathrm{dB}(\mu \mathrm{V} / \mathrm{m})]$ \\
\hline $30-230$ & 40 \\
\hline $230-1000$ & 47 \\
\hline
\end{tabular}

Table 3. The limits for radiated emissions for above 1 $\mathrm{GHz}^{1}$

\begin{tabular}{|l|l|l|}
\hline $\begin{array}{l}\text { Frequency } \\
{[\mathrm{GHz}]}\end{array}$ & Average $[\mathrm{dB}(\mu \mathrm{V} / \mathrm{m})]$ & $\begin{array}{l}\text { Quasi-peak } \\
{[\mathrm{dB}(\mu \mathrm{V} / \mathrm{m})]}\end{array}$ \\
\hline $1-3$ & 56 & 76 \\
\hline $3-6$ & 60 & 80 \\
\hline
\end{tabular}




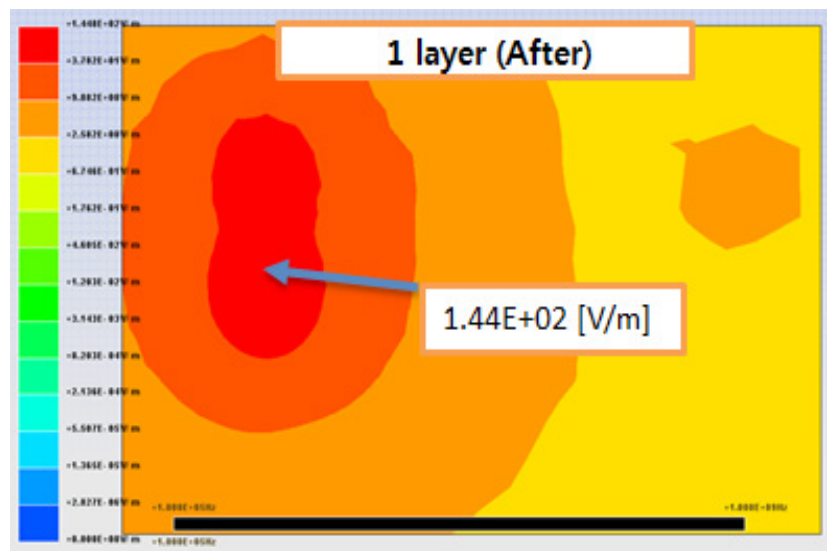

Figure 11. 1-layer near field mode test (E-field).

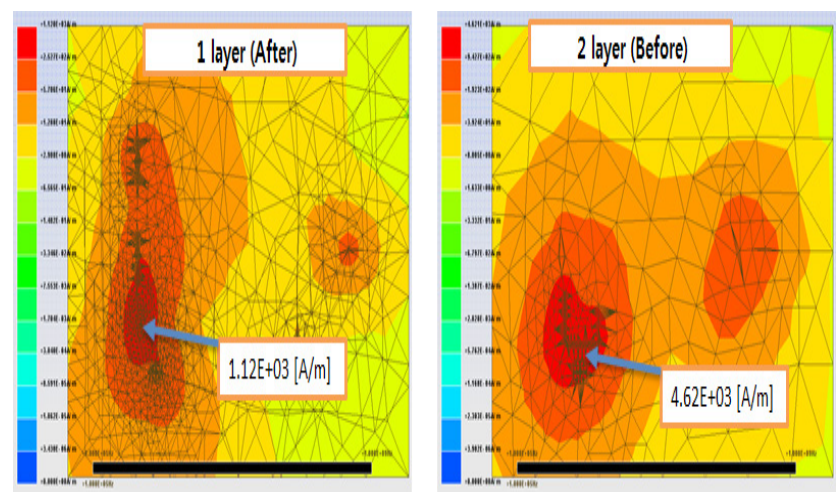

Figure 12. 2-layer near field mode test (H-field) before applying EMI reduction techniques.

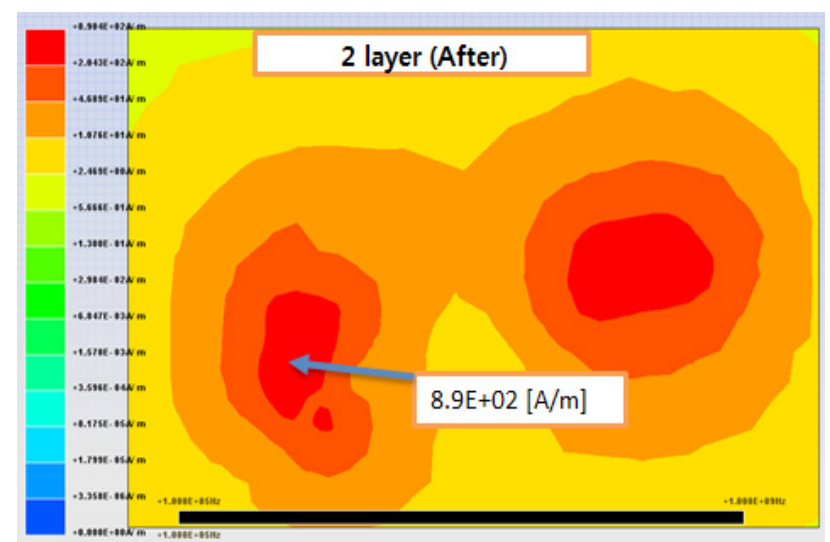

Figure 14. 2-layer near field mode test (H-field) after applying EMI reduction techniques.

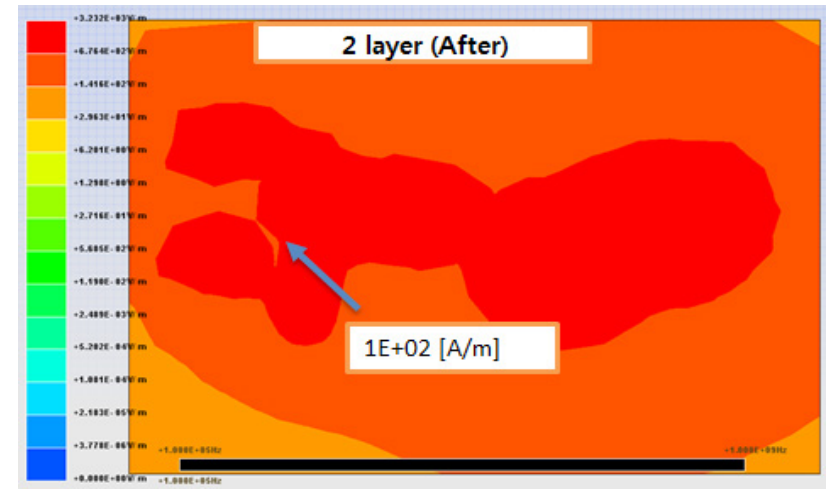

Figure 15. 2-layer near field mode test (E-field) after applying EMI reduction techniques.

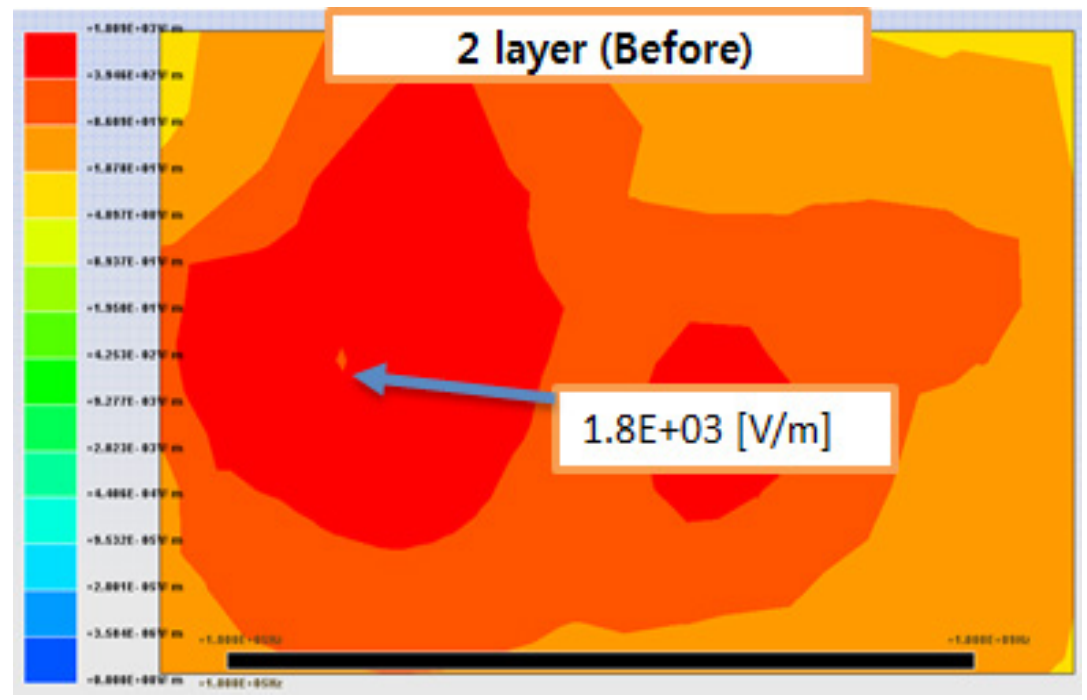

Figure 13. 2-layer near field mode test (E-field) before applying EMI reduction techniques. 

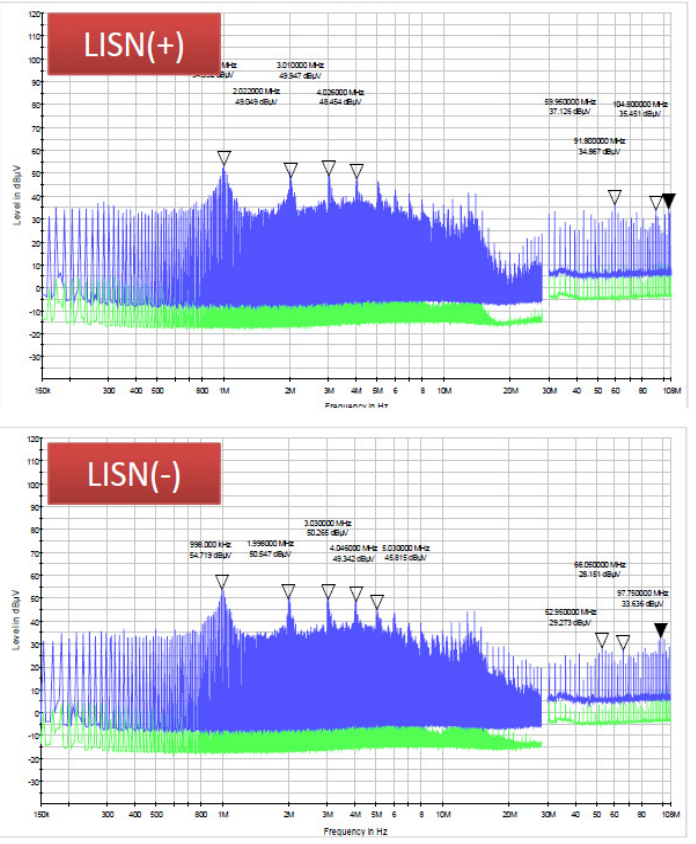

Figure 16. CISPR-22 conducted RF emissions (+, - Voltage on supply lines).

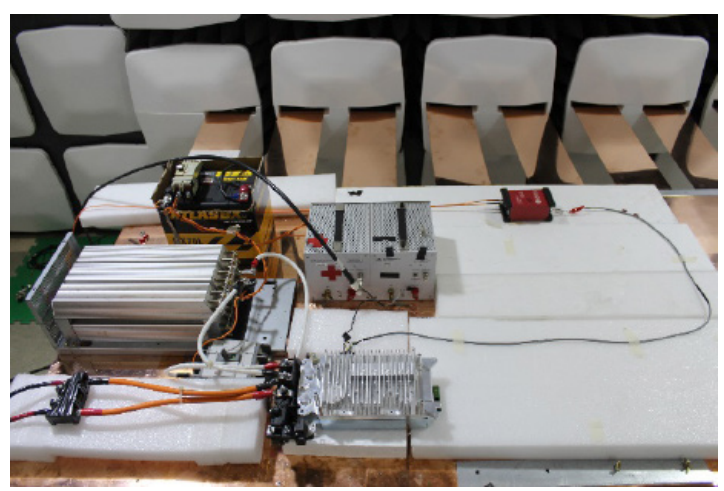

Figure 17. CISPR-22 conducted RF emissions test setup.

\section{Conclusions}

In this study, we present an investigation study on EMI reduction techniques of a power converter circuit for solar energy system. A DC-DC power converter circuit is designed and manufactured. The simulation results are presented. To reduce the unwanted emissions, an optimized circuit with capacitors and ground posts is manufactured. Measurement method and conditions used here are suggested. Conducted and radiated emission were measured based on CISPR-22 standard. By using 2-layered circuit, unintentional emissions are diminished tremendously.
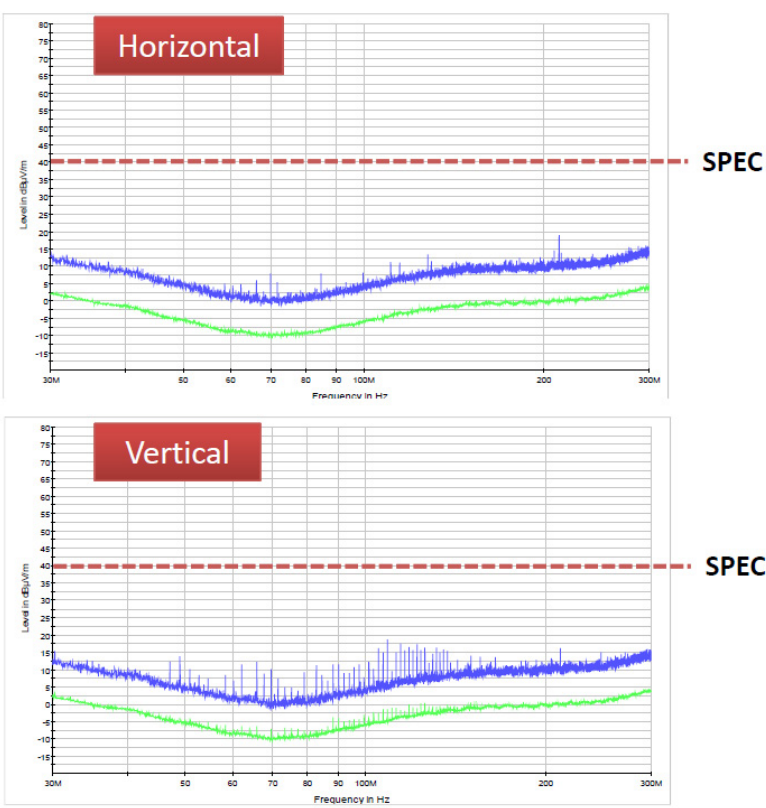

Figure 18. CISPR-22 radiated RF emissions (horizontal and vertical antenna position).

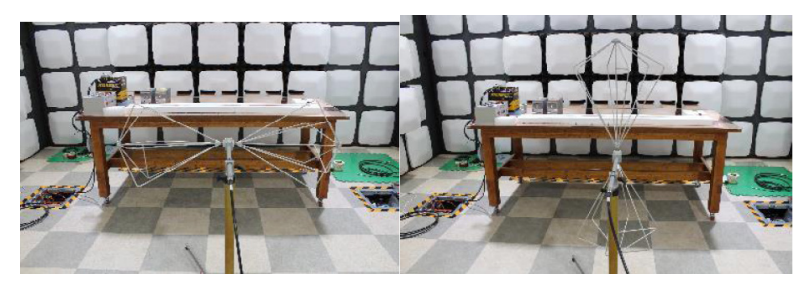

Figure 19. CISPR-22 radiated RF emissions setup.

\section{Acknowledgement}

This work was supported by 2016 Baekseok University research fund. The author would like to thank Jae-Young Yeo, CEO of NEST, for his guide on CISPR standards and valuable help to get the test results used here.

\section{References}

1. Paul C. Introduction to Electromagnetic Compatibility. 2nd ed. Wiley-Interscience; 2006 Jan.

2. International Electro Technical Commission. 2005. Available from: https://webstore.iec.ch/p-preview/info_ cispr22\%7Bed5.0\%7Den_d.pdf

3. Radio disturbance characteristics for the protection of receivers used on board vehicles, boats and on devices - Limits and methods of measurement. 2002. Available from: https://webstore.iec.ch/p-preview/info cispr25\%7Bed2.0\%7Den_d.pdf 
4. Rodriguez V. Automotive component EMC testing: CISPR 25, ISO 11452-2 and equivalent standards. IEEE Electromagnetic Compatibility Magazine. 2012; 1(1):8390.

5. Zare F. EMI issues in modern power electronic systems. The IEEE EMC Society Newsletters. 2009; 221:53-8.

6. Thiagu G, Dhanasekaran R. Design of low EMI power supply using resonant converter. Indian Journal and Science and Technology. 2015 Jun; 8(12):1-6.

7. Karthik B, Kiran Kumar TVU. EMI developed test methodologies for short duration noises. Indian Journal and Science and Technology. 2013 May; 6(S5):1-5.

8. Basics of Noise Countermeasures [Lesson 6] Common mode choke coils. 2011. Available from: http://www. murata.com/products/emiconfun/emc/2011/10/28/en20111028-p1

9. A generic automotive EMC Test Standard. 2006. Available from: http://www.autoemc.net/Papers/Test/ OHaraGenericEMCStd.pdf

10. Nadolny J. Testing for radiated emission by electronic devices. Association for Molecular Pathology Journal of Technology. 1995 Jun; 4:111-5.
11. Measurement Comparisons of Radiated Test Facilities. 1992. Available from: http://ieeexplore.ieee.org/document/626124/

12. Simulating Open Area Test Site Emission Measurements Based on Data Obtained in a Novel Broadband TEM Cell. 1989. Available from: http://ieeexplore.ieee.org/document/37174/

13. Balanis CA. Antenna theory analyis and design. 3rd ed. Wiley-Interscience; 2005 Apr.

14. Pozar DM. Microwave ENGINEERIng. 4th Revised ed. Wiley; 2011 Dec.

15. Bean JL, Hall RA. Electromagnetic susceptibility measurements using a mode stirred chamber. Proceedings of International Symposium on Electromagnetic Compatibility (A79-25301 09-32); Atlanta, New York: Institute of Electrical and Electronics Engineers, Inc; 1978 Jun. p. 143-50. 